\title{
D-ZERO END CAP CALORIMETER ANNULAR PIPING ANALYSIS
}

D-Zero Engineering Note: 3740.220-EN-175

C.H. Kurita

$\begin{array}{lll}\text { August } \quad 22, & 1988\end{array}$

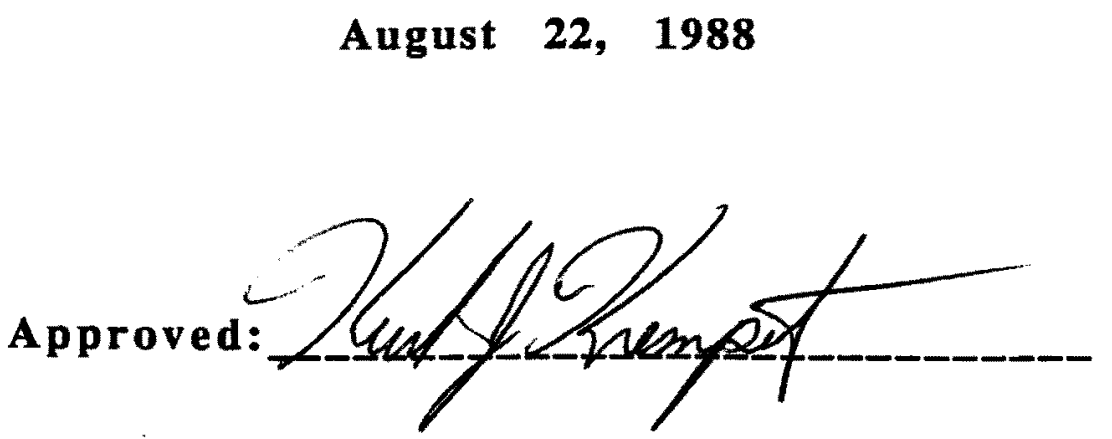


In accordance with the ASME Code for Pressure Piping, B31 version of Chemical Plant and Petroleum Refinery Piping. ANSI/ASME B31.3-1984 Edition, the maximum allowable stress for 304 stainless steel piping is 25,500 psi. This "Allowable Displacement Stress Range" value was calculated using equation (1a) found on page 16 of the above mentioned reference.

Each of the lines that comprise the End Cap Calorimeter (EC) piping were modeled on ANSYS and the appropriate constraints were applied. This was done using the Piping Stress Analysis Module of ANSYS. The bending stresses and displacements due to the thermal contraction that occurs in cooling the piping from $300 \mathrm{~K}$ to $77 \mathrm{~K}$ were calculated by ANSYS. The seven lines involved and their maximum bending stresses are as follows:

\section{LINE}

1) Rupture Disc

2) Argon Relief

3) Gaseous Argon Supply

4) Liquid Nitrogen Cooldown Supply

5) Liquid Nitrogen Operating Supply

6) Nitrogen Exhaust

7) Argon/Nitrogen Vent
MAX. BEND. STRESS

8335 psi

12,619 psi

5399 psi

7049 psi

5774 psi

1826 psi

48,364 psi

The maximum bending stress values and their positions are indicated on the attached diagrams. The dashed line indicates the original position of the piping, and the solid line shows the position of the displaced piping. Also attached are copies of the programs used to model the piping configurations. The original analysis was done by $J$. Wendlandt, but later changes and additions required that the programs be modified and re-run. The maximum bending stress found in each line of the annular piping fell sufficiently below the allowed maximum of $25,500 \mathrm{psi}$. The maximum bending stress for the Argon/Nitrogen Vent line was $48,364 \mathrm{psi}$, which is greater than the value of $25,500 \mathrm{psi}$ allowed by the code. This line of piping is located on the outside of the annular space and will be installed at Fermilab. While fabrication of the EC vessel is underway, modifications will be made to the Argon/Nitrogen Vent line in order that its stress values remain within that allowed by the code.

The complete Argon/Nitrogen Vent line is analyzed in EN-312, DØ Cryogenic Piping Frame Stress Analysis, and the stresses are below the maximum allowable stress of 25,500 psi. 


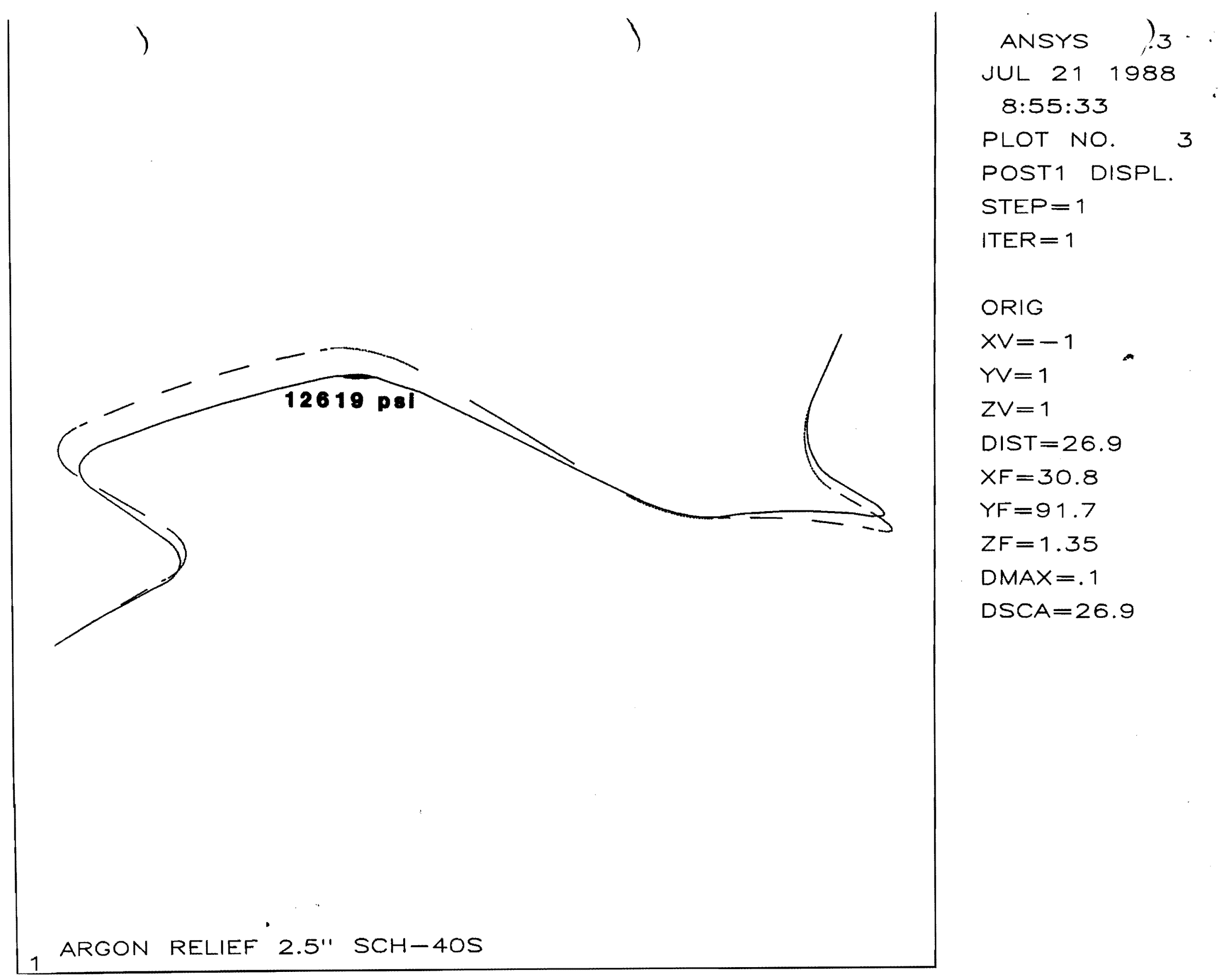




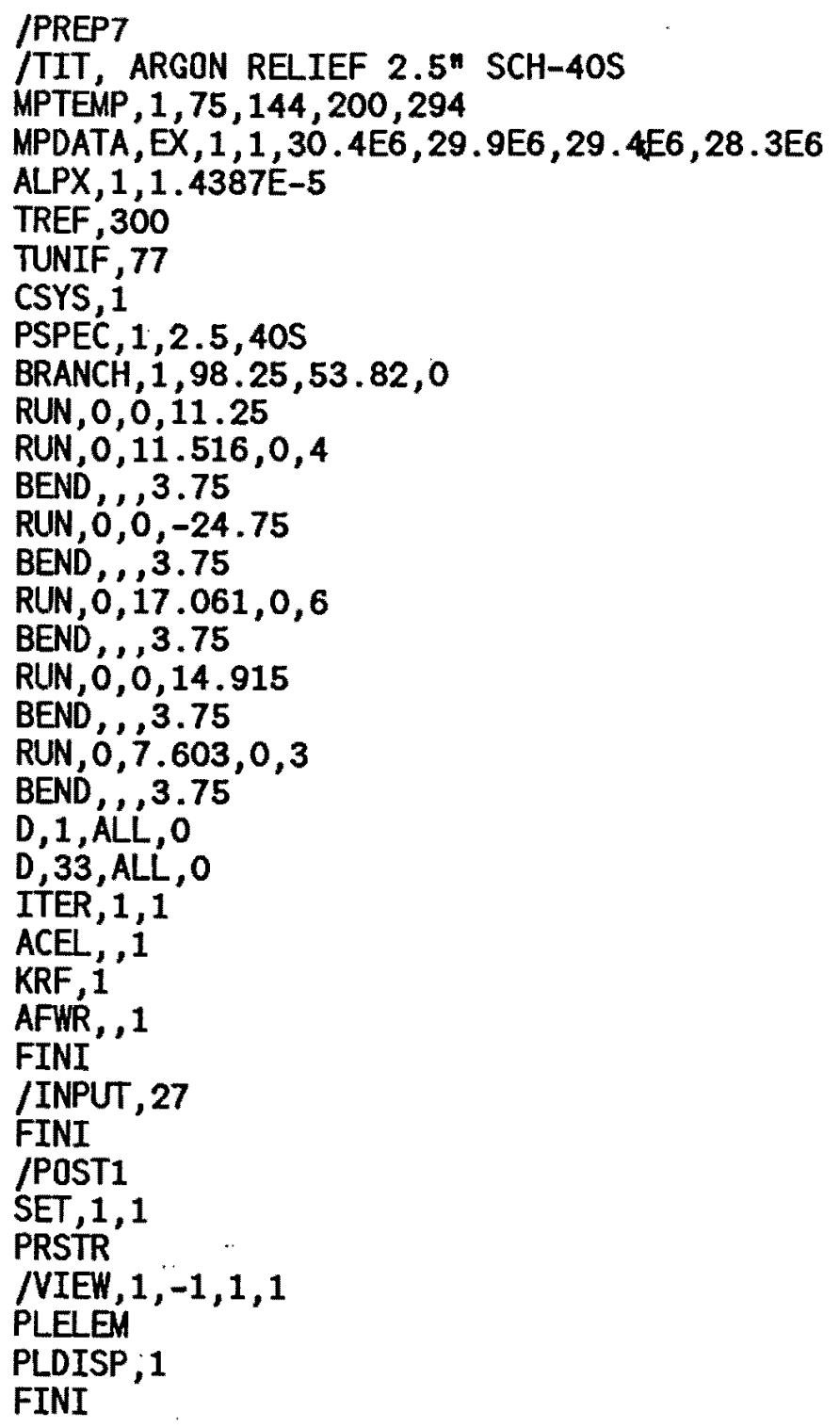




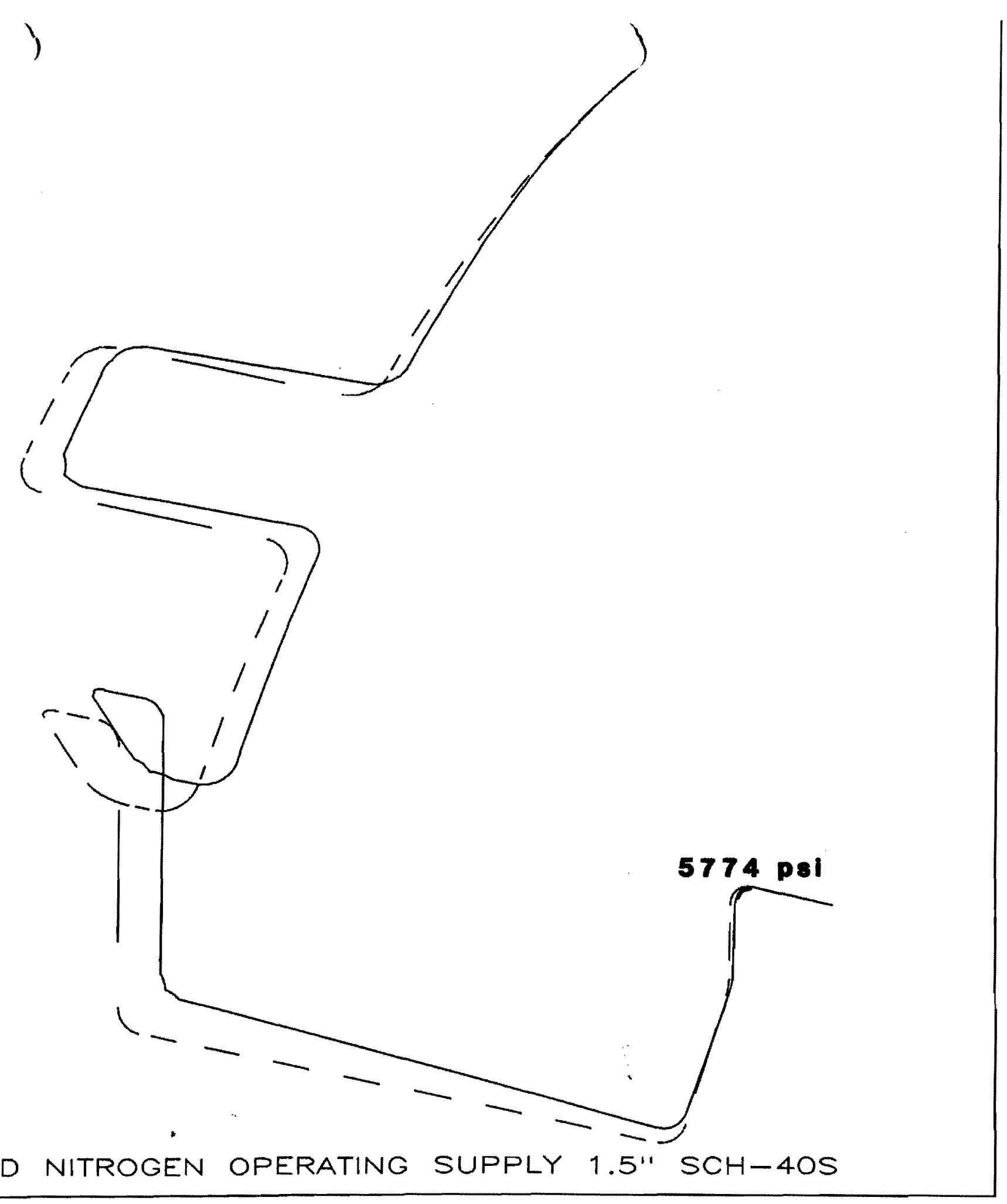
$\begin{array}{lr}\text { ANSYS } & 3 \\ \text { JUL } 25 & 1988\end{array}$ 7:56:38 PLOT NO. 3 POST1 DISPL. STEP $=1$ ITER $=1$

ORIG $X V=-2$ $Y V=1$ $Z V=1$ $\mathrm{DIST}=37$ $X F=-45.1$ $Y F=80.7$ $Z F=7.45$ $D M A X=.229$ $D S C A=16.1$ 
/PREP7

/TIT, LIQUID NITROGEN OPERATING SUPPLY $1.5^{\mathrm{n}}$ SCH-40S

MPTEMP , 1, 75, 144, 200, 294

MPDATA, EX, 1, 1, 30.4E6, 29.9E6, 29 . 4₹6, 28.3E6

ALPX, 1, 1. 4387E-5

TREF, 300

TUNIF, 77

CSYS, 1

PSPEC $1,1.5,40 \mathrm{~S}$

BRANCH, 1, 98, 90,0

RUN, $0,22.931,0,8$

RUN, $0,0,-21$

BEND, , 2.25

RUN, $0,7.126,0,3$

BEND, , , 2.25

RUN, $0,0,21$

BEND, 2.25

RUN, $0,11.884,0,5$

BEND, ,, 2.25

RUN, $0,0,4.042$

BEND, , 2.25

$D, 1, A L L$

$D, 36$, ALL

ITER, 1,1

ACEL, , 1

KRF,1

AFWR, , 1

FINI

/INPUT, 27

FINI

/POST1

SET, 1,1

PRSTR

IVIEW , 1,-1, 1, 1

PLELEM

PLDISP ; 1

FINI 


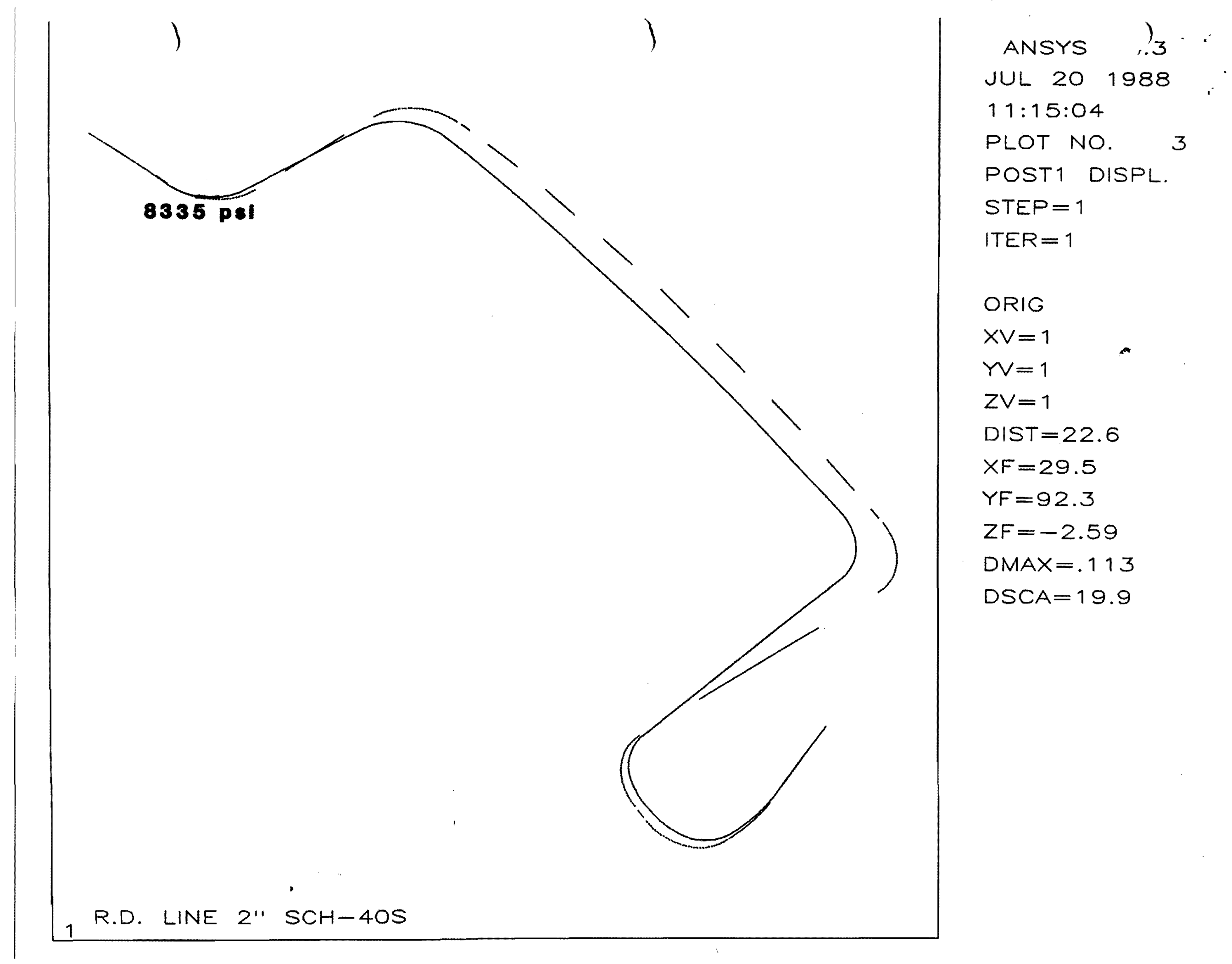


/PREP7

/TIT, R.D. LINE 2" SCH-40S

MPTEMP , 1, 75, 144, 200, 294

MPDATA, EX, 1, 1 , 30.4E6 , 29 . 9E6, 29 . 4E6 , 28. 3E6

ALPX, $1,1.309 E-5$

TREF, 300

TUNIF, 77

CSYS, 1

PSPEC , 1, 2, 40S

BRANCH, $1,107.678,58.641,0$

RUN, $-9.678,0,0$

RUN, $0,0,5.25$

BEND, , , 2

RUN , 0, 4.026, 0

BEND, , , 3

RUN, $0,0,-23.0$

BEND, , , 3

RUN, $0,22.065,0,9$

BEND, , , 3

RUN, $0,0,14.6875$

BEND, , , 3

RUN, $0,5.269,0,2$

BEND, , , 3

$D, 1, A L L$

$D, 37, A L L$

ITER , 1,1

ACEL, 1

KRF, 1

AFWR, , 1

FINI

/INPUT, 27

FINI

/POST1

SET, 1, 1

PRSTR

PRDIS

FINI 


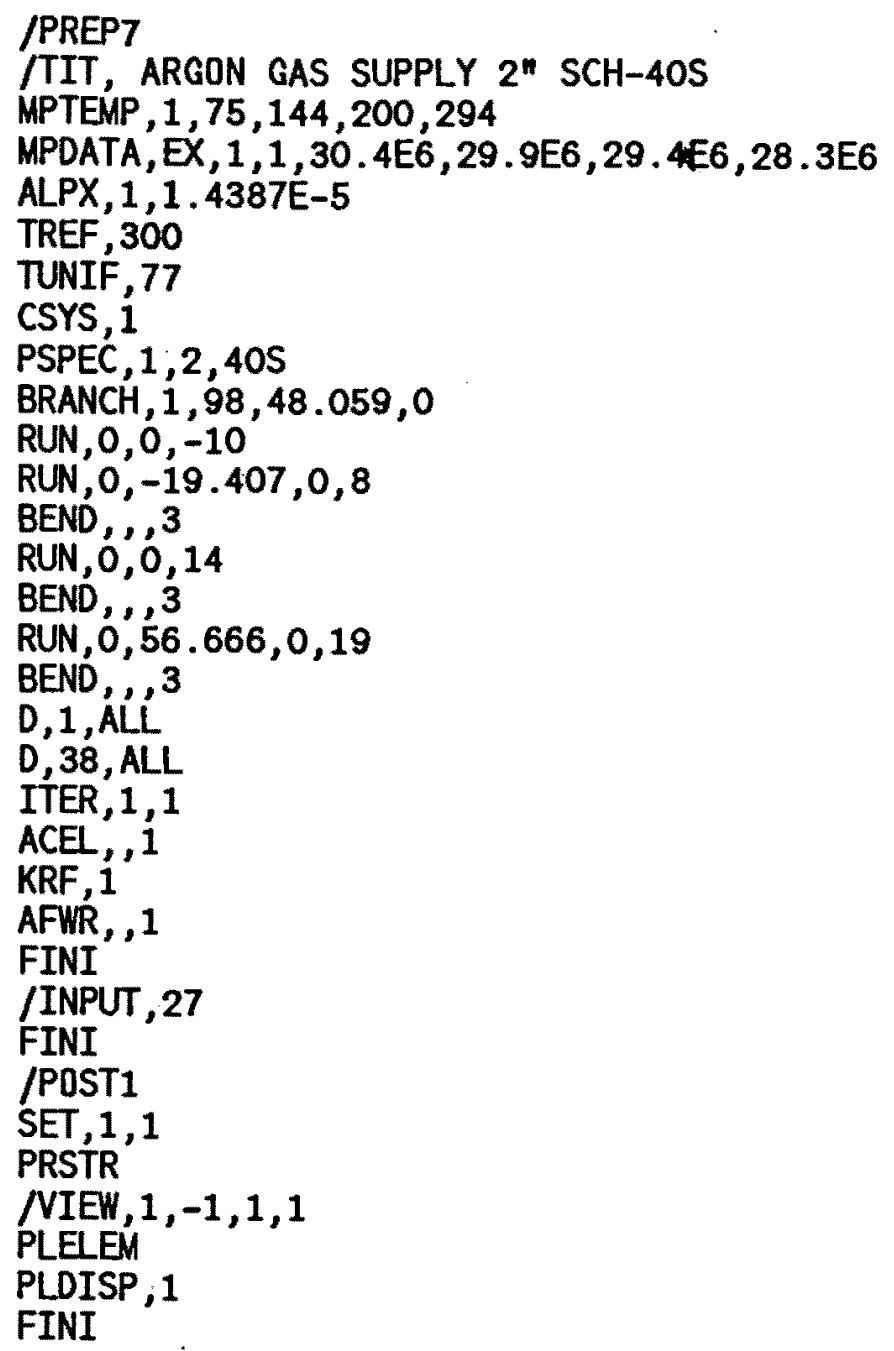




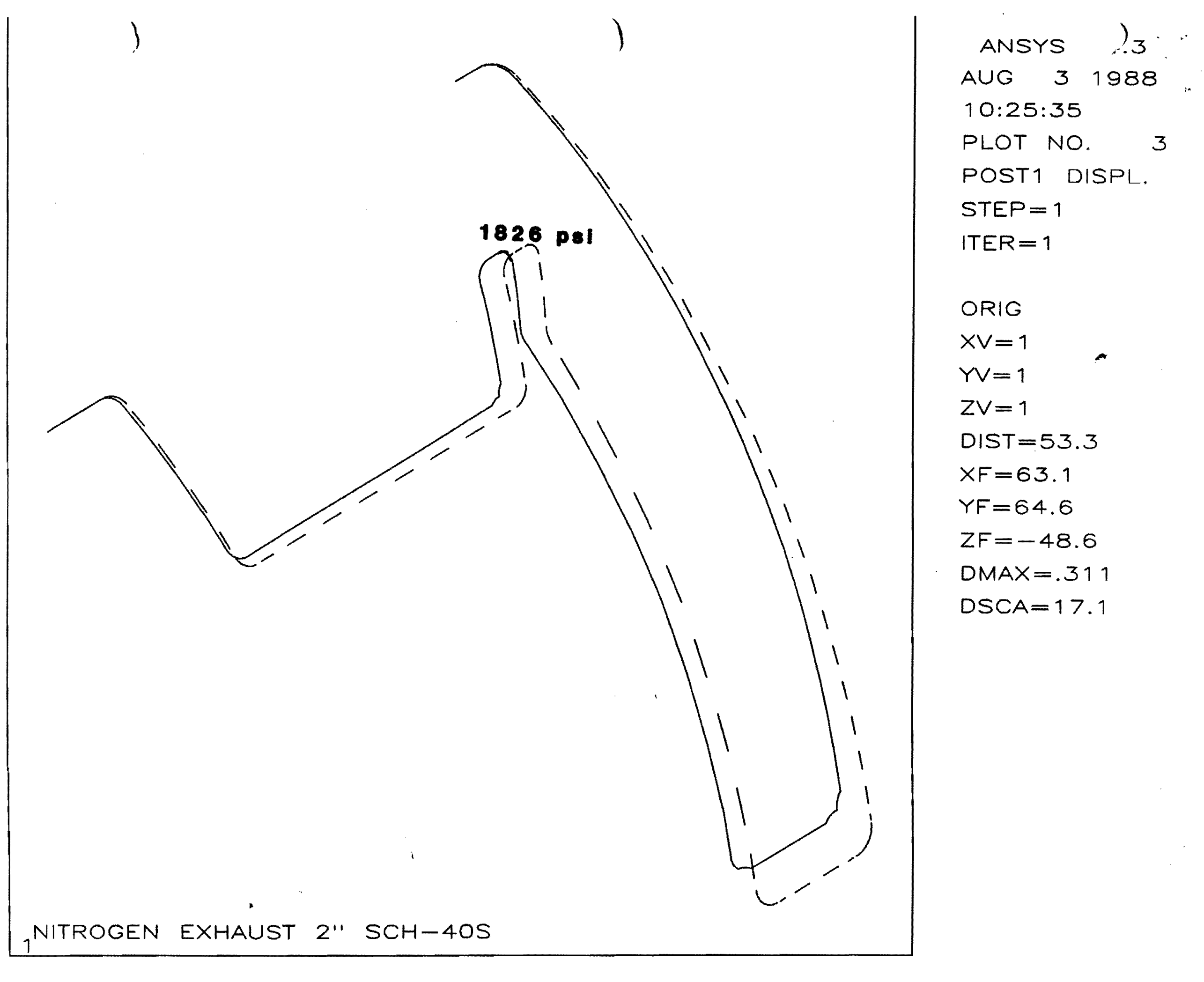


/PREP7

/TIT NITROGEN EXHAUST 2 " SCH-40S

MPTEMP , 1, 75, 144, 200, 294

MPDATA, EX, 1, 1, 30 .4E6, 29.9E6, 29 . 4E6 , 28 . 3E6

ALPX, 1, 1.309E-5

TREF, 300

TUNIF, 77

CSYS, 1

PSPEC, 1, 2, 10S

BRANCH, $1,96.444,63.364,0$

RUN, $0,0,-11.35$

RUN, $0,-15.277,0,4$

BEND, , , 2

RUN , $0,0,-47.463,8$

BEND, , , 2

RUN, 10.547,7.946, 0,3

BEND, , , 2

RUN, $0,0,-6$

BEND, , 2

PSPEC $1,2,40 S$

RUN, $-8.991,-4.902,0,2$

BEND, , , 2

RUN, $0,-42.931,0,8$

BEND, , , 3

RUN, $0,0,-19,4$

BEND, , ,3

RUN, $0,61.348,0,24$

BEND, , , 3

RUN, $0,0,7.0625$

BEND, , 3

$D, 1, A L L$

$D, 89, A L L$

ITER, 1,1 .

ACEL, , 1

KRF, 1

AFWR, 1

FINI

/INPUT, 27

FINI

/POST1

SET , 1,1

PRSTR

FINI 


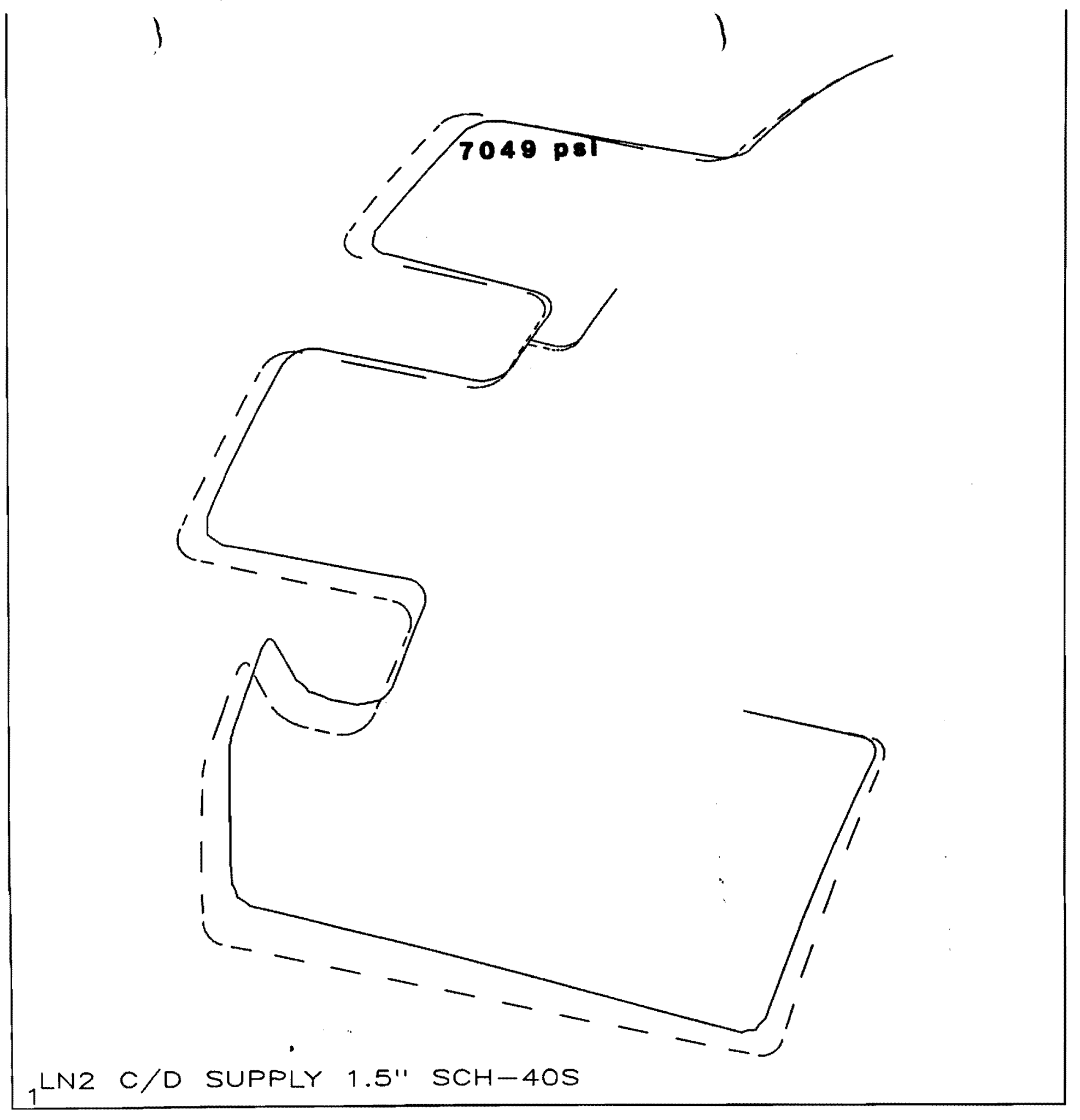

ANSYS $!_{3}$. AUG 31988 10:33:25 PLOT NO. 3 POST1 DISPL. $S T E P=1$ ITER $=1$

ORIG $X V=-2$ $Y=1$ $Z V=1$ $\mathrm{DIST}=38.6$ $X F=-35.6$ $Y F=80.1$ $Z F=-.124$ $\mathrm{DMAX}=.194$ $D S C A=19.9$ 
/PREP7

/TIT,LN2 C/D SUPPLY $1.5^{n} \mathrm{SCH}-40 \mathrm{~S}$

MPTEMP , 1, 75, 144, 200, 294

MPDATA, EX, 1, 1, 30.4E6, 29.9E6, 29 .4E6, 28.3E6

ALPX, 1, 1.309E-5

TREF, 300

TUNIF, 77

CSYS, 1

PSPEC $1,1.5,40 S$

BRANCH, $1,98,76.818,0$

RUN , 0,16.16,0,7

RUN, $0,0,-21.750$

BEND, , 2.25

RUN, $0,11.666,0,5$

BEND, , 2.25

RUN, $0,0,18$

BEND, , , 2.25

RUN, $0,3.043,0$

BEND, , 2.25

RUN , 0, 3.095, 0

RUN, $0,0,-18$

BEND, , 2.25

RUN, $0,11.577,0,5$

BEND, ,, 2.25

RUN, $0,0,20.6875,5$

BEND, , 2.25

RUN, $0,6.564,0,3$

BEND, ,, 2.25

RUN, $0,0,-6.9375$

BEND, , , 2.25

RUN, $9.825,0,0$

BEND, , , 2.25

PSPEC, 1, 1.5, 10S

RUN, 0, 4.782,0

BEND, , , 1.5

RUN, $-9.125,5.297,0,3$

BEND, , 1.5

RUN, $0,0,46.8125,10$

BEND, , , 1.5

RUN , $0,-14.730,0,5$

BEND, , 1.5

RUN , $0,0,-12,3$

BEND, , 1.5

BRANCH, 28

RUN, $0,0,3.75$

TEE, , , 1.3,1.3,1.3

RUN, $0,-4.434,0,2$

BEND, , 1.5

$D, 1, A L L$

D,111,ALL

$D, 121, A L L$

ITER, 1, 1

ACEL, , 1

KRF, 1

AFWR, , 1

FINI

/INPUT, 27

FINI

/POST1

SET , 1, 1

PRSTR

FINI 


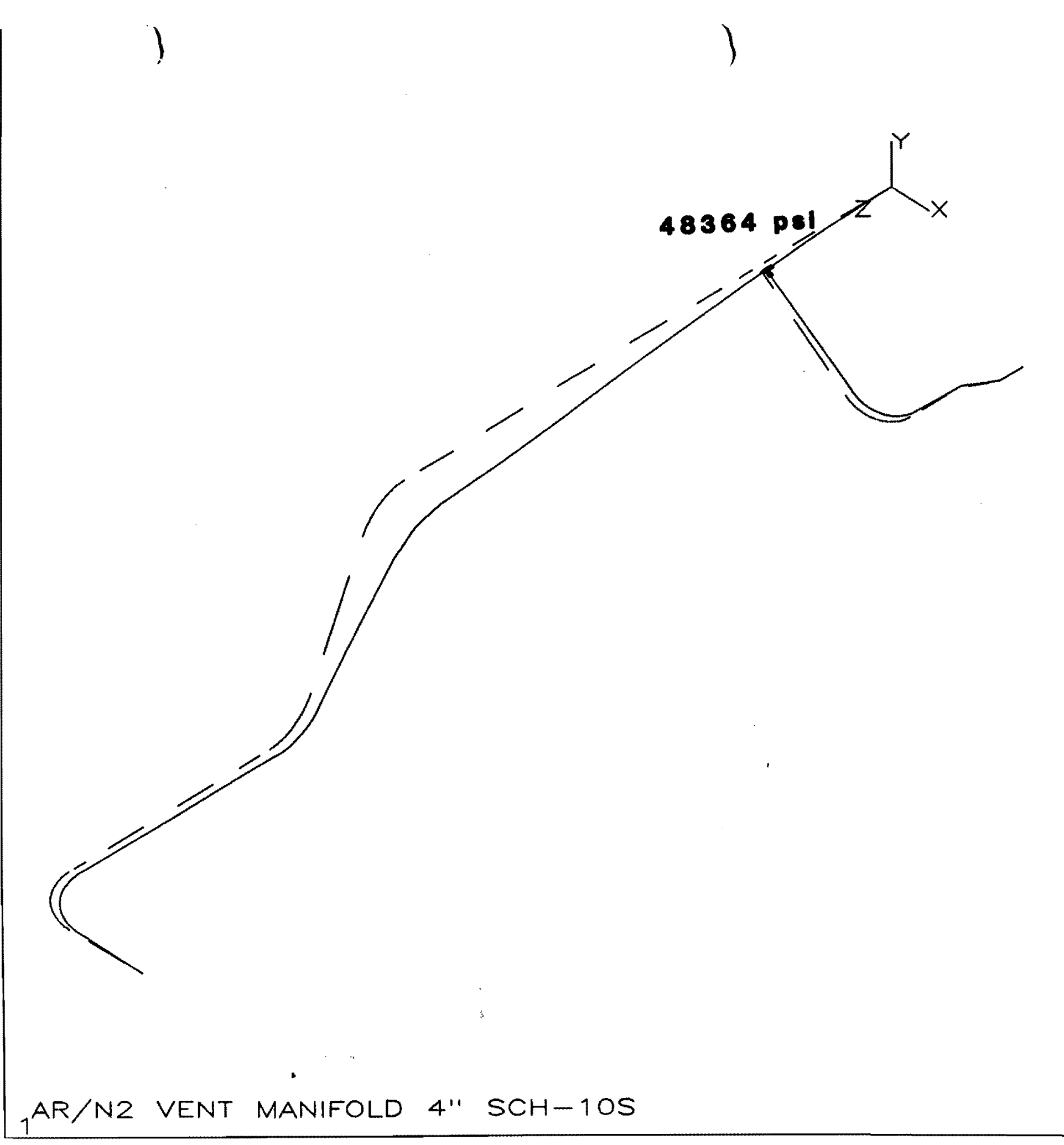

ANSYS $\quad .3$ AUG 31988 9:46:00 PLOT NO. 1 POST1 DISPL. $S T E P=1$ ITER $=1$

ORIG

$X V=1$

$Y V=1$

$Z V=1$

$D I S T=31.6$ $X F=5.82$

$Y F=-6.47$

$\mathrm{ZF}=35.6$

$D M A X=.182$ $\mathrm{DSCA}=17.3$ 


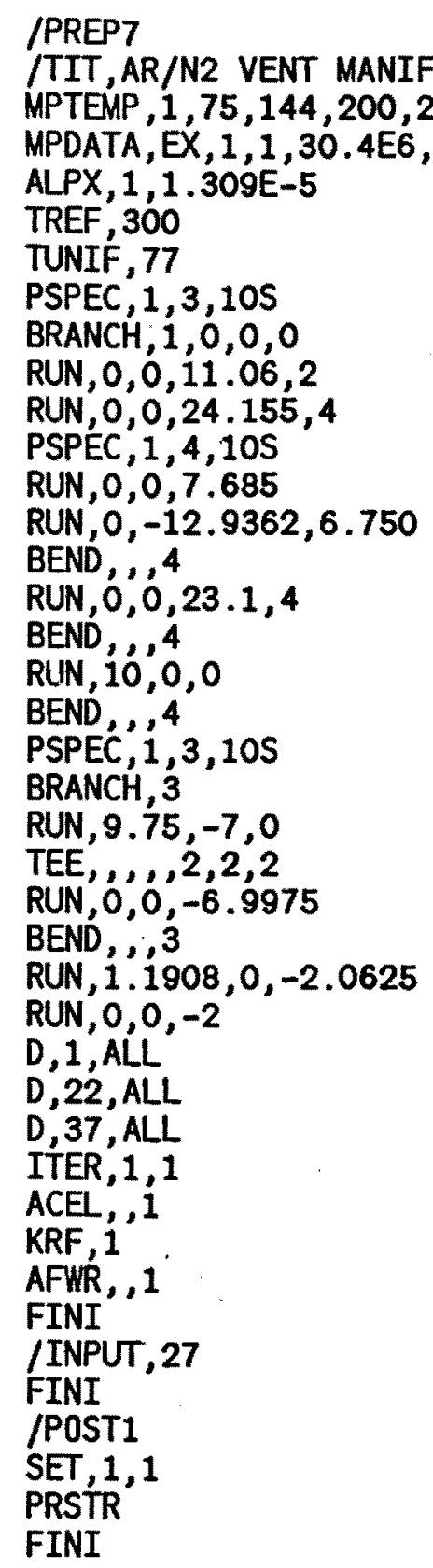

